\title{
Liquid-crystal electro-optic modulator based on electrohydrodynamic effects
}

\author{
M. A. Muriel and J. A. Martin-Pereda \\ Departamento de Electrónica Cuántica, E.T.S. Ingenieros de Telecomunicación, Ciudad Universitaria, Madrid-3, Spain
}

Received June 17, 1980

\begin{abstract}
A new method of light modulation is reported. This method is based on the electro-optical properties of nematic materials and on the use of a new wedge structure. The advantages of this structure are the possibility of modulating nonpolarized light and the improved signal-to-noise ratio. The highest modulating frequency obtained is 25 $\mathrm{kHz}$.
\end{abstract}

In this Letter we report a new method of light modulation based on the electro-optic properties of nematic liquid crystals. The basis of this method is the use of a wedged structure not previously reported for this application. The advantages of such a structure, as we will show later, are based mainly on the signal-to-noise ratio, which is better than with other configurations. The importance of the first property is based on possible applications in which a polarization-insensitive device can be required. The second property derives from the wedged structure used, which permits a total separation between rays.

Our modulator has two main features: (a) the use of nematic liquid crystal, with $\Delta \epsilon<0$, as the anisotropic medium; (b) the use of a rather special structure, which is a wedge in which the orientation of the nematic liquid-crystal molecules is parallel to the vertex.

As is well known, ${ }^{1,2}$ nematic liquid crystals exhibit a unique behavior. Depending on the frequency and voltage applied, two regimes appear. For frequencies lower than a certain cutoff frequency, $f_{c}$, the space charge in the fluid oscillates at the same frequency as the driving signal. This regime is known as the conduction regime. When the applied frequency is greater than $f_{c}$, the space charge does not oscillate. The fluid interacts with the applied field to result in a dielectric regime. The threshold field is proportional to $f^{1 / 2}$. This field, contrary to that in the low-frequency situation, is thickness independent. Periodic deformations, known as chevrons, result from the field-fluid interaction. The spatial frequency of the chevron striation is a monotonically increasing function of the driving frequency. Moreover, according to the voltage-versusfrequency representations of these two regimes, it is necessary to take into account the fact that the limiting frequency to maintain the chevron pattern, for a given voltage, is dependent on the cell thickness, $d$. The relation is

$$
V \propto d f^{1 / 2} .
$$

Hence the only limiting factor in the possible applications of these patterns is the thickness of the cell, the common value being larger than $10 \mu \mathrm{m}$. With this cell thickness, for a square wave with $100-\mathrm{V}$ peak voltage, the frequency is around $150 \mathrm{~Hz}$ (cutoff frequency near $100 \mathrm{~Hz}$ ).
Otherwise, in the dielectric regime, also known as the fast-turnoff mode, if the ac voltage is turned off from a value above a certain threshold voltage to zero, the chevrons disappear rapidly. According to de Gennes, ${ }^{1}$ the relaxation rate is

$$
1 / T \simeq \kappa q^{2} / \eta,
$$

where $\kappa$ is the Frank elastic constant, $q$ is the spatial frequency of striations, and $\eta$ is the viscosity coefficient.

Since $q$ is proportional to $f^{1 / 2}$, the relaxation rate will be proportional to the applied frequency. This is analogous to the creation of standing waves in an elastic medium. ${ }^{3}$

If optical radiation propagates transversely through a planar sandwich cell with chevron structure, the motion of these domains does not strongly affect the propagation direction of such radiation. This can be shown theoretically as well as empirically.

Moreover, if unpolarized light goes through the structure described above, the emerging signal will be decomposed into two orthogonally polarized components. The one that keeps the same direction as the incident light is the ordinary ray with a polarization perpendicular to the initial direction of the molecules. The second one will be the extraordinary ray polarized orthogonally to the preceding one. If the molecular situation corresponds to a Williams mode, the first beam will appear as a spot but the second one will be split into several spots corresponding to the diffraction pattern of the Williams domains. ${ }^{4}$ If the regime is dynamic scattering, just two spots appear, one for each of the two possible rays, ordinary and extraordinary. No diffraction pattern will appear. If the sandwich cell presents a chevron mode, both rays will give diffraction patterns. In every case, the zero-order diffraction positions for both rays will be very close to each other and indistinguishible without polarizers.

From the above considerations, if some information is impressed upon the applied voltage between electrodes, this information will be difficult to detect. This is so because of the spatial coincidence of both rays in the detector. Otherwise, if a polarizer is used, some intensity will be lost.

To overcome the above limitations, we have devel- 
oped a structure consisting of a wedge cell with a Mylar spacer on one side. This structure has been used by other workers in this field for the measurement of refraction indices ${ }^{5,6}$ and for the separation of ordinary and extraordinary rays..$^{7}$ Our wedge angle was about $2^{\circ}$, and the separation at the opposite side of the Mylar spacer was $4 \mu \mathrm{m}$. The incident ray, after crossing the wedge, will be split into the two components previously indicated, but with a substantial modification. These two components will be clearly separated in space and in this way will be easier to distinguish. Both components will travel forming angles $\alpha_{o}$ and $\alpha_{e}$ with the incidence direction. Their values are

$$
\begin{aligned}
& \alpha_{o}=\sin ^{-1}\left(n_{o} \sin \alpha\right)-\alpha, \\
& \alpha_{e}=\sin ^{-1}\left(n_{e} \sin \alpha\right)-\alpha,
\end{aligned}
$$

with $n_{o}$ and $n_{e}$ the ordinary and extraordinary refraction indices, respectively, and $\alpha$ the wedge angle (Fig. 1 ). The angle difference is given by

$$
\Delta \alpha=\sin ^{-1}\left(n_{o} \sin \alpha\right)-\sin ^{-1}\left(n_{e} \sin \alpha\right) .
$$

For small values of $\alpha$, as in our case, this becomes

$$
\Delta \alpha \simeq \alpha\left(n_{e}-n_{o}\right)=\alpha \Delta n .
$$

If the observation plane is at a distance $D$ away from the cell, the linear separation between both zero-order diffraction positions will be

$$
\Delta z \simeq \alpha D \Delta n \text {. }
$$

With this structure, we have avoided, as we indicated before, the use of polarizers and, moreover, because of the very small distance between electrodes near the vertex of the wedge, it is possible to work at much higher frequencies than those previously obtained. 8,9

The results of our measurements are shown in Fig. 2. We have used Licristal Phase 5, supplied by Merck, with a nematic phase temperature range from $-5^{\circ} \mathrm{C}$ to $75^{\circ} \mathrm{C}$. Our working temperatures were $20^{\circ} \mathrm{C}$ and $50^{\circ} \mathrm{C}$. We used an unpolarized $0.5-\mathrm{mW} \mathrm{He}-\mathrm{Ne}$ laser as the light source. The measurements were performed with a conventional silicon solar-cell photodetector placed at the zero-order diffraction position of the extraordinary ray. The ordinary ray was excluded. As can be seen from Fig. 2, two regimes appear. The first one goes from 0 to $100 \mathrm{~Hz}$, the second one from the first frequency to $25 \mathrm{kHz}$. Because the modulating signal was a 40 -V-rms pulsed voltage, the first region corresponds to a dynamic scattering mode. The second region corresponds to a chevron configuration. The modulation is obtained, in this situation, from the energy

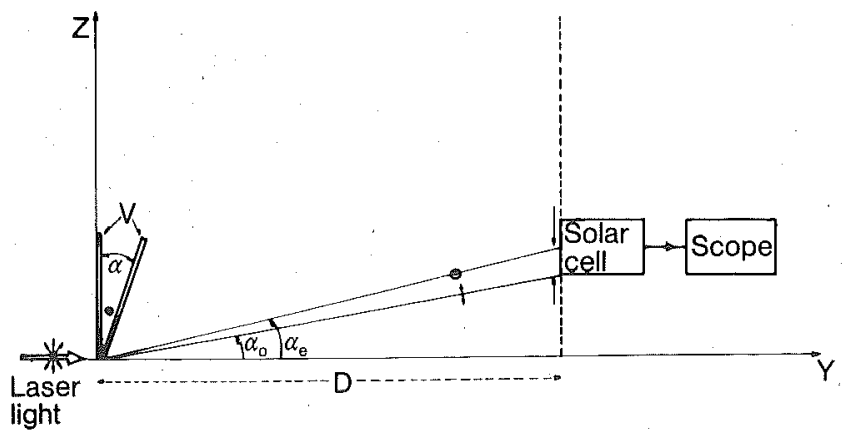

Fig. 1. Geometrical configuration for the modulation effect.

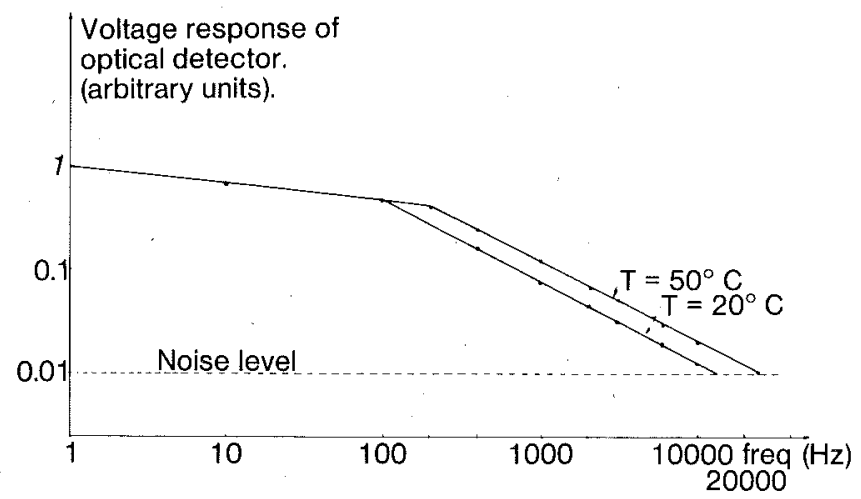

Fig. 2. Frequency response of the homogeneous wedge structure.

transfered to the higher-order diffraction rays. The optical modulation depth at $f=1 \mathrm{~Hz}$ was $45 \%$ of the unpolarized input beam. This modulation depth begins to roll off at $100 \mathrm{~Hz}$ and decreases with a slope of $7.7 \mathrm{~dB}$ per decade, which results in a modulation depth of $0.5 \%$ at $f=25 \mathrm{kHz}$. The bandwidth of the detection system used was $60 \mathrm{kHz}$.

This $25-\mathrm{kHz}$ modulation was heretofore impossible to obtain in a chevron sandwich cell with square or sine waves.

This wedge structure can be used too with liquidcrystal materials with $\Delta \epsilon>0$, but we have not yet performed the corresponding experiments. A digital light-beam deflector and an analog light-beam deflector, using electric fields, have been reported by us ${ }^{10,11}$ for these types of material. The configuration was similar, but instead of the homogeneous texture we adopted a twisted molecular configuration in the first case and a homogeneous texture, perpendicular to the vertex, in the second one. Moreover, similar results have been obtained by us with magnetic fields. This proves the usefulness of our configuration and its applications in this field.

We acknowledge the support provided by the Spanish Comisión Asesora de Investigación Científica y Técnica.

\section{References}

1. P. G. deGennes, The Physics of Liquid Crystals (Oxford U. Press, London, 1974), Chap. V.

2. S. Chandrasekhar, Liquid Crystals (Cambridge U. Press, London, 1977), Chap. III.

3. G. H. Conners and K. B. Paxton, J. Appl. Phys. 43, 2959 (1972).

4. T. O. Carroll, J. Appl. Phys. 43, 767 (1972).

5. I. Haller, H. A. Huggins, and M. J. Freiser, Mol. Cryst. Liq. Cryst. 16, 53 (1972).

6. P. Chatelain and M. Germain, C. R. Acad. Sci. Paris 259, 127 (1964).

7. S. Sato and A. Kikuchi, Oyo Butsuri 45, 938 (1976).

8. H. Hacker et al., Appl. Opt. 19, 1278, (1980).

9. Y. S. Kwon, I. Lefkowitz, and R. Lontz, Appl. Opt. 18, 1700 (1979).

10. M. A. Muriel and J. A. Martín-Pereda, presented at 1980 European Conference on Optical Systems and Applications, Utrecht, September 1980.

11. M. A. Muriel and J. A. Martín-Pereda, presented at 1980 Annual Meeting of the Optical Society of America, Chicago, October 1980. 